Recepción: 21 / 04 / 2018

Aceptación: 13 / 05 / 2018

Publicación: 02 / 07 / 2018

Ciencias de la Salud

Artículo de Investigación

\title{
Incidencia de las TIC en la formación de los Terapistas Ocupacionales
}

\section{Incidence of ICT in the training of Occupational Therapists}

\section{Incidência das TIC na formação dos Terapistas Ocupacionales}

\author{
Edison L. Barcia-Carrillo ${ }^{\mathrm{I}}$ \\ barcaele@hotmail.com \\ Mar Camacho II \\ mar.camacho@urv.cat
}

Correspondencia: barcaele@ hotmail.com

\footnotetext{
${ }^{\text {I }}$ Diploma Superior en Educación Universitaria por Competencias, Analista de Sistemas, Ingeniero en Sistemas, Docente de la Universidad Laica “Eloy Alfaro” de Manabí.

II Docente, Universitat Rovira i Virgili, Departamento de Pedagogía, Tarragona, España.
} 


\title{
Resumen
}

El objetivo del presente artículo, se corresponde a constatar los conocimientos sobre TIC en docentes y estudiantes y su grado de integración durante el proceso enseñanza aprendizaje en la formación de los futuros licenciados en Terapia Ocupacional de la Universidad Laica "Eloy Alfaro" de Manabí. Se aplicó una metodología cuali-cuantitativa, bajo un diseño no experimental y de tipo descriptivo y como instrumento de recolección de la información un cuestionario electrónico. Entre los resultados se pudo constatar que el $100 \%$ del total de los investigados coincidieron en opinar que conocían sobre sistemas operativo y los aplican, además utilizaban laptop. Entre las conclusiones se evidenció que los docentes y alumnos tienen conocimiento general de herramientas informáticas, en las que se identificó el uso adecuado del Sistema Operativo y del Software de Ofimática en el proceso de planificación y diseño de actividades académicas en la impartición de clases.

Palabras clave: TIC; tecnología educativa; Terapia Ocupacional; recursos didácticos y tecnológicos.

\begin{abstract}
The aim of this article is to verify the knowledge about ICT in teachers and students and their degree of integration during the teaching-learning process in the training of future graduates in Occupational Therapy of the "Eloy Alfaro" University of La Manabí. A qualitative-quantitative methodology was applied, under a non-experimental and descriptive design and as an instrument for collecting the information, an electronic questionnaire. Among the results it was possible to verify that $100 \%$ of the total of the investigated ones coincided in opining that they knew about operating systems and they apply them, also they used laptop. Among the conclusions, it was evidenced that teachers and students have general knowledge of computer tools, in which the proper use of the Operating System and the Software of Ofimatics in the process of planning and designing academic activities in the teaching of classes was identified.
\end{abstract}

Keywords: ICT; educational technology; Occupational therapy; didactic and technological resources. 


\section{Resumo}

O objetivo do presente artigo, se corresponde a um processo de aprendizagem sobre TIC em estudantes e estudiosos de integração durante o processo enseñanza aprendizado na formação de futuros licenciados em Terapia Ocupacional da Universidade Laica "Eloy Alfaro" de Manabí. Se aplicou uma metodologia cuali-cuantitativa, não possui nenhum instrumento experimental e descritivo como instrumento de recuperação da informação e informação eletrônica. Entre os resultados obtidos, consta que $100 \%$ do total dos investigadores coincidiu em relação ao conceito de sistemas operacionais e aplicativos, usando um laptop. Entre as conclusões se evidenciam que os docentes e ex-alunos se conformam em geral de informática, nos quais são identificados o uso do Sistema Operativo e do Software de Ofimática no processo de planificação e desenho de actividades académicas na comunicação de clases.

Palavras chave: TIC; tecnología educativa; Terapia Ocupacional; recursos didáticos e tecnológicos.

\section{Introducción}

El compromiso de los gobiernos y organizaciones, debe manifestarse en la búsqueda de mecanismos que permitan la incorporación de las Tecnologías de la información y la comunicación en la educación, garantizando el acceso a las nuevas formas de enseñanza al potenciar la disponibilidad de los recursos computacionales (UNESCO, 2013), sin embargo es importante comprender que a pesar que los diseños curriculares reflejan estas herramientas tecnológicas en su planificación, aún existe un alto porcentaje de incidencias que determinan una carencia de integración de las múltiples bondades de los sistemas informáticos como herramienta interactiva en la cátedra (Ramírez, Casillas, Contreras, 2014); esta carencia es percibida en la resistencia de algunos integrantes de la planta docente, en relación del uso de la tecnología o en otros casos el utilizarla de forma elemental al evidenciar que, únicamente han cambiado las láminas tradicionales de acetato por las digitales, denotando la no incursión en la gran diversidad de formatos de información disponibles en la red (Correa, Fernández, Gutiérrez, Lozada, Ochoa, 2015), también es importante reconocer que esta problemática podría estar ligada tanto a la dependencia del modelo tradicional, como a la falta de mejoras de políticas de igualdad de oportunidades (Lugo, Schurmann, Schurmann, 2012). 
Sin embargo, la demanda emergida por las exigencias computacionales de un contexto que genera constantes "golpes" hacia el quehacer educativo en todos los niveles y que se relacionan con la necesidad de adquirir innovaciones tecnológicas, que se califican como impostergables para aquellas instituciones que desean continuar activas y reconocidas como de alto desempeño, esta particularidad permite que se evidencie al detenerse por un momento a visualizar las características de los centros de formación y con ello se confirma el crecimiento del número de establecimientos que incorporan el uso de las Tecnologías de las Información y Comunicación, tanto en la gestión docente como en la administrativa (Henríquez, 2011). A tal efecto, en este estudio se pudo constatar los conocimientos sobre TIC en docentes y estudiantes y su grado de integración durante el proceso enseñanza aprendizaje de la carrera de Terapia Ocupacional de la Universidad Laica "Eloy Alfaro" de Manabí.

\section{Desarrollo}

Mora (2009) en su libro: "La interfaz hipermedia: El paradigma de la comunicación interactiva", analiza como las instituciones educativas y culturales han tenido que despertar, educar y evolucionar al estudio de los nuevos medios de expresión, de igual forma refiere el uso de un modelo comunicativo de hipermedia eficiente que produzca creaciones lúdicas-educativas con la pretensión de que los docentes puedan aprovechar estos recursos interactivos a favor de los estudiantes e incluso aplicarlos como estrategia en aquellos que presenten diferencias cognitivas, perceptivas o físicas.

Los sistemas de comunicación basados en la informática, que integran la interactividad y la diversidad de medios expresivos multisensoriales, aspiran al desarrollo de una inmersión comunicativa (fisiológica, psicológica y emocionalmente) tan intensa como la experimentada con la realidad sensible no binaria. (p. 27).

Es importante que docentes y estudiantes incorporen en su rol la aplicación de la Tecnología de la Información y Comunicación con un tinte de innovación que aproveche los recursos y bondades provenientes del Hardware y Software disponible creando un ambiente de disfrute en la acción de aprehender.

En este sentido, Salinas (2000), indica que "El énfasis se debe de hacer en la docencia, en los cambios de estrategias didácticas de los profesores, en los sistemas de comunicación y 
distribución de materiales de aprendizaje, en lugar de enfatizar la disponibilidad y las potencialidades de las tecnologías" (p67).

Asimismo, un dato interesante es el descrito por Monreal, Giráldez y Gutiérrez (2015) en su artículo: "Uso e Integración Curricular de la Pizarra Digital Interactiva (PDI) en la Clase de Música en Primaria: Un Estudio de Caso en Segovia” en donde enfatiza en su estudio que el clima del aula, grado de motivación e interés del alumnado lo marca el profesor y la actitud positiva de los alumnos ante nuevos aprendizajes, además se intensifica la atención de los estudiantes cuando son ellos los partícipes del proceso académico.

En el contexto de formación de los futuros profesionales en Terapia Ocupacional se manifiestan circunstancias en las que aplicaciones de escritorio y online permiten mejorar notablemente su desarrollo académico, sin embargo, es de destacar que existen muchos otros programas informáticos diseñados con características de accesibilidad que pueden convertirse en una valiosa ayuda en la intervención de personas con algún grado de discapacidad.

Ciertamente es de resaltar por ejemplo, lo propuesto por la UNESCO (2016), quienes apuntan que organizaciones internacionales y gobiernos han realizado considerables incorporaciones para mejorar las características educacionales a favor de los niños con necesidades educativas especiales, sin embargo aún no es suficiente, es evidente el ritmo trepidante de los avances tecnológicos con novedosas particularidades que deben ser configurados de forma adecuada (Mora, 2009) y los cuales podrían responder a estímulos generados por los sentidos del ser humano intensificando su atención en el aula (Rubio, 2004), confirmando entonces que la herramienta tecnológica es una firme candidata para ingresar al grupo nuevos paradigmas interactivos como estrategia activa que busca garantizar una educación inclusiva y equitativa (UNESCO, 2016).

A tal efecto, se hace fundamental comprender la importancia de cambiar el paradigma de aquellos educadores que se resisten parcial o totalmente al uso de la tecnología, existen muchas investigaciones que han demostrado que el uso de la tecnología puede ser parte de un cambio de modelo educativo que magnifica las oportunidades de aprendizaje y en este caso, los futuros terapistas ocupacionales tendrían un plus que redundaría en su desempeño profesional a favor de quienes requieran de sus servicios. 
Ante esta posición, el alumno será “el agente de su propia educación por medio de la interacción permanente de sus acciones y su reflexión" (Pérez, 2001, p.45), en este caso los estudiantes demuestran un gran dominio de terminología informática, conocimiento de programas y uso de dispositivos móviles, esto es aún más visible cuando acceden a internet desde sus domicilios o incluso por la red inalámbrica de la institución en donde los educandos mantienen una interconectividad constante que maximiza las oportunidades de cumplir con las exigencias de estudio.

Asimismo, Marqués (2000) afirma que indiscutiblemente el desarrollo tecnológico crea nuevos paradigmas de enseñanza dando pauta a tener nuevas metodologías, roles, perfiles docentes, gestiones académicas y administrativas en nuevos entornos de aprendizaje.

\section{Metodología}

En la presente investigación, se aplicó un diseño no experimental y una metodología cualicuantitativa, de tipo descriptivo con el fin de captar información sobre la invidencia de las TIC en la formación de los terapistas ocupacionales siendo parte de la investigación mixta y no experimental que según Gómez (2010), corresponde a "representar un escenario que ya viene dado, no obstante, se pueda distinguir valores para evaluar relaciones entre variables" (p.87).

\section{Resultados}

Posterior a la información obtenida, por el instrumento aplicado fue posible presentar los siguientes resultados.

Tabla 1. Conocimiento de TIC en los docentes

\begin{tabular}{|l|c|c|c|c|}
\hline \multicolumn{1}{|c|}{ Conocimientos } & Si & $\mathbf{\%}$ & No & \% \\
\hline Sistema Operativo & 30 & 100,0 & 0 & 0,0 \\
\hline Software de ofimática & 30 & 100,0 & 0 & 0,0 \\
\hline Correo electrónico & 30 & 100,0 & 0 & 0,0 \\
\hline Aula virtual & 30 & 100,0 & 0 & 0,0 \\
\hline Empaquetadores & 5 & 16,7 & 25 & 83,3 \\
\hline Edición de video & 0 & 0,0 & 30 & 100,0 \\
\hline Almacenamiento en la nube & 8 & 26,7 & 22 & 73,3 \\
\hline Redes sociales & 25 & 83,3 & 5 & 16,7 \\
\hline
\end{tabular}

Fuente: Cálculos del autor. 
Se observa en la tabla 1, el análisis descriptivo de la información obtenida acerca de los conocimientos sobre las TIC de los docentes y estudiantes, en la misma se indica que el 100\% del total de los investigados coincidieron en opinar que conocían sobre sistemas operativos, software de ofimática, correo electrónico y aula virtual. Seguida del 83,3\% para las redes sociales, un $26,7 \%$ en almacenamiento en la nube y el 16,7 solo conocían acerca de las empaquetadoras. Se destaca el hecho, que no hubo manifestación de conocimiento para la edición de videos.

Tabla 2. Aplicación de las TIC

\begin{tabular}{|l|c|c|c|c|}
\hline \multicolumn{1}{|c|}{ TIC y docencia } & Si & \% & No & $\%$ \\
\hline Aplica TIC en la docencia & 30 & 100,0 & 0 & 0,0 \\
\hline $\begin{array}{l}\text { Ha aprendido nuevos programas } \\
\text { en los últimos años }\end{array}$ & 4 & 13,3 & 26 & 86,7 \\
\hline
\end{tabular}

Fuente: Cálculos del autor.

La tabla 2, presenta el análisis descriptivo de la información obtenida acerca de la aplicación de los TIC por parte de los docentes y estudiantes, en la misma se indica que el $100 \%$ del total de los investigados, coincidieron en opinar que si la aplican y solo el 13,3\% manifestaron que han aprendido nuevos programas en los últimos años.

Tabla 3. Tipos de equipos que utilizan

\begin{tabular}{|l|c|c|c|c|}
\hline \multicolumn{1}{|c|}{ Tipo de equipos que utiliza } & $\mathbf{S i}$ & $\mathbf{\%}$ & $\mathbf{N o}$ & $\mathbf{\%}$ \\
\hline PC & 5 & 16,7 & 25 & 83,3 \\
\hline Laptop & 30 & 100,0 & 0 & 0,0 \\
\hline Tablet & 2 & 6,7 & 28 & 93,3 \\
\hline Teléfono inteligente & 5 & 16,7 & 25 & 83,3 \\
\hline
\end{tabular}

Fuente: Cálculos del autor.

En la tabla 3, se presenta el análisis descriptivo de la información obtenida acerca de los tipos de equipos que utilizan por parte de los docentes y estudiantes, en la misma se indica que el 100\% del total de los investigados coincidieron en opinar que utilizaban laptop, el 16,7\% por igual utilizan PC y teléfono inteligente. El menor porcentaje en opiniones fue para el uso de Tablet con el $6,7 \%$ del total de ellos. 


\section{Conclusiones}

En cuanto a la constatación de los conocimientos sobre TIC en docentes y estudiantes de la carrera de Terapia Ocupacional de la Universidad Laica "Eloy Alfaro" de Manabí, se pudo determinar que:

Los docentes y alumnos tienen conocimiento general de herramientas informáticas, en las que se identificó el uso adecuado del Sistema Operativo y del Software de Ofimática en el proceso de planificación y diseño de actividades académicas en la impartición de clases.

Entre las fortalezas existentes en la planta docente, es que todos cuentan con equipos portátiles en donde realizan las actividades docentes y de gestión, estos equipos tienen instalado Microsoft Windows como sistema operativo en versiones que varían a partir desde la identificada como Seven, como paquete de ofimática prevalece la suite Microsoft Office desde la versión 2013.

Los docentes y estudiantes, hacen uso del correo institucional que es entregado a cada profesor por la Unidad Central de Coordinación de Informática de la institución y que debe utilizar para comunicarse con estudiantes y autoridades, de igual forma lo referente a la organización de contenidos, publicación de notas, asistencia, material didáctico o recolección de trabajos se lo efectúa mediante el uso del aula virtual de la IES en la dirección aulavirtual.uleam.edu.ec.

Fue posible evidenciar, que los estudiantes poseen un mayor conocimiento de las aplicaciones de escritorio y web que son utilizadas para el diseño de las tareas enviadas por lo docentes y/o aquellas relacionadas a las prácticas pre-profesionales lo que posibilita ambientes de autoeducación y confirmación de conocimientos.

Se determinar que a pesar de que los salones de clase tienen disponibilidad de una computadora de escritorio y un proyector, los docentes preparan material didáctico en software de ofimática, mientras que los estudiantes demuestran dominio de otras herramientas, lo que genera una integración limitada estancada en procesos computarizados rutinarios ubicados en el uso de archivos PDF, DOC y PPT, que son suficientes para abarcar las temáticas de estudio pero en los estudiantes queda la insatisfacción del no uso de otros formatos de información con los que se rompan esquemas y se visualice un novedoso modelo educativo basado en las bondades existentes en el avance de los sistemas computarizados. 
Se corroboró el uso de programas tradicionales, pero también la incorporación de herramientas de comunicación formal e informal, entre los más utilizados se destacan: aula virtual, email, Facebook, chats, videoconferencias, grupos de Whatsapp, almacenamiento en la nube, Google Drive, empaquetadores y otras herramientas de trabajo colaborativo que son incorporadas de forma efectiva en el proceso enseñanza aprendizaje y que son parte inexcusable de la educación 2.0.

Fue posible deducir, que los docentes de las asignaturas de informática entregan un conocimiento teórico práctico de carácter transversal y por tanto es reutilizado por todas las asignaturas que forman la malla curricular del terapista ocupacional, sin embargo los demás docentes que dictan asignaturas básicas y profesionales pueden apoyarse en software especializado que capte la atención de los estudiantes y facilite el aprendizaje, aquí se podrían destacar las enciclopedias en $3 \mathrm{~d}$, aplicaciones de registro, control y seguimiento de pacientes, simuladores de casos de estudio, software para ordenadores adaptados como teclados virtuales, programas de reconocimiento de voz, lectores de pantalla, magnificadores de pantalla, sintetizadores de voz, reconocedor óptico de caracteres, entre otros.

\section{Referencias Bibliográficas}

Correa J., Fernández L., Gutiérrez A., Losada D., Ochoa B. (2015). "Formación del profesorado. Tecnología Educativa e Identidad Docente Digital”. Revista Latinoamericana de Tecnología Educativa. Volumen 14. Número 1, mayo 2015. Disponible en: http://relatec.unex.es/article/view/1841/1370

Gómez, D. R., \& Roquet, J. V. (2010). Metodología de la investigación. México: Red Tercer Milenio. Revista Publicando, 4 No 10.(1). 2017, 458-474. ISSN 1390, 9304473.

Henríquez, P.M. (2011): La aplicación didáctica de las tecnologías de la informática y la comunicación en la formación del siglo XXI. Tarragona, Facultad de Educación, tesis doctoral inédita.

Lugo M., Schurmann V., Schurmann S. (2012). "Políticas TIC en educación en América Latina: Más allá del modelo 1:1”. Revista Científica de Tecnología Educativa. Volumen 1. Número 1, 
agosto

2012.

Argentina.

Disponible

en:

http://uajournals.com/ojs/index.php/campusvirtuales/article/view/17/16

Marqués, P. (2000). La cultura tecnológica en la sociedad de la información. Entornos Educativos. Departamento de Pedagogía Aplicada. Universidad Autónoma de Barcelona. Recuperado el, 31(03), 2015.

Monreal, I, Giraldez, A, Gutierrez, A. (2015). "Uso e Integración Curricular de la Pizarra Digital Interactiva (PDI) en la Clase de Música en Primaria: Un Estudio de caso en Segovia. Artículo publicado en REMIE - Multidisciplinary Journal of Educational Research. Volumen 5. Número 1, febrero 2015 pp. 82-104. Disponible en:

https://dialnet.unirioja.es/servlet/articulo?codigo=4994733

Mora Fernández, J. (2009). La interfaz hipermedia: El paradigma de la comunicación interactiva. Madrid: Ediciones y publicaciones Autor S.R.I. Disponible en: http://www.academia.edu/5684744/La_Interfaz_Hipermedia_El_paradigma_de_la_Comunicaci\% C3\%B3n_Interactiva

Pérez Serrano, G. (2001). Aprender a lo largo de la vida: desafío de la sociedad actual.

Ramírez, A., Casillas, M., Contreras, C. (2014). La incorporación de las TIC a la enseñanza universitaria de los idiomas. Artículo publicado en la Revista Académica electrónica Debate Universitario de la Universidad Abierta Interamericana. Volumen 3. Número 5, noviembre de 2014. Disponible en: http://portalreviscien.uai.edu.ar/ojs/index.php/debateuniversitario/article/view/20/24

Rubio Molina, P, Santoyo Velasco, C. (2004). "Interacciones sociales de niños con necesidades educativas especiales". Artículo publicado en el Portal de revistas Científicas y Arbitradas de la UNAM. Volumen 30. Número 1, marzo de 2004 México. Disponible en: http://www.revistas.unam.mx/index.php/rmac/article/view/25201/23690

Salinas, J. (2000). El aprendizaje colaborativo con los nuevos canales de comunicación. Síntesis, Madrid, 199-228. 
UNESCO (2013). "Uso de TIC en la Educación en América Latina y el Caribe: Análisis regional de la integración de las TIC en la educación y de la aptitud digital (e-readiness). Disponible en: http://unesdoc.unesco.org/images/0021/002193/219369s.pdf

UNESCO (2016). La educación al servicio de los pueblos y el planeta: Creación de futuros sostenibles para todos.

http://unesdoc.unesco.org/images/0024/002457/245745s.pdf 